\title{
Article
}

\section{M-polynomial of some cactus chains and their topological indices}

\author{
Bommanahal Basavanagoud ${ }^{1, *}$ and Anand P. Barangi ${ }^{1}$ \\ 1 Department of Mathematics, Karnatak University, Dharwad - 580003, Karnataka, India.; \\ b.basavanagoud@gmail.com(B.B); apb4maths@gmail.com(A.P.B) \\ * Correspondence: b.basavanagoud@gmail.com
}

Received: 15 April 2019; Accepted: 21 May 2019; Published: 29 July 2019

Abstract: In this note, we first show that the general Zagreb index can be obtained from the $M$-polynomial of a graph by giving a suitable operator. Next, we obtain $M$-polynomial of some cactus chains. Furthermore, we derive some degree based topological indices of cactus chains from their $M$-polynomial.

Keywords: M-polynomial, general Zagreb index, cactus chain.

MSC: 05C31.

\section{Introduction}

$\mathbf{T}$ he concept of degree is analogous to the concept of valence in organic chemistry with a limitation that degree of any vertex in any chemical graph is at most 4 . This gives graph theory a broad way to chemistry. It is always interesting to find some properties of graphs or molecular graphs which are invariant. Topological indices and polynomials are foremost among them. Over the last decade there are numerous research papers devoted to topological indices and polynomials. Several topological indices have been defined in the literature. For various topological indices one can refer to [1-8].

Let $G=(V, E)$ be a simple, undirected graph. Let $V(G)$ be the vertex set and $E(G)$ be the edge set of the graph $G$, respectively. The degree $d_{G}(v)$ of a vertex $v \in V(G)$ is the number of edges incident to it in $G$. Let $\left\{v_{1}, v_{2}, \ldots, v_{n}\right\}$ be the vertices of $G$ and let $d_{i}=d_{G}\left(v_{i}\right)$. A graph $G$ is said to be $r$ - regular if degree of each vertex in $G$ is $r$. A graph is called cycle if it is 2- regular. A cactus graph is a connected graph in which any two simple cycles have at most one vertex in common. Every cycle of cactus graph is chordless and every block of a cactus graph is either an edge or a cycle. If all blocks of a cactus graph are triangular then it is called triangular cacti. If all the triangles of a triangular cactus graph has at most two cut-vertices and each cut-vertex is shared by exactly two triangles then we say that triangular cactus graph is a chain triangular cactus. In chain triangular cactus if we replace triangles by cycles of length 4 then we obtain cacti whose every block is $C_{4}$, such cacti are called square cacti. For ortho-chain square cactus the cut vertices are adjacent and a para-chain square cactus their cut vertices are not adjacent. Recent study on some cactus chain can be found in [9-11] and references cited therein. For undefined graph theoretic terminology used in this paper can be found in [12].

The general form of degree-based topological index of a graph is given by

$$
T I(G)=\sum_{e=u v \in G} f\left(d_{G}(u), d_{G}(v)\right)
$$

where $f=f(x, y)$ is a function appropriately chosen for the computation. Table 1 gives the standard topological indices defined by $f(x, y)$.

The $M$ - polynomial [13] was introduced in 2015 by Deutch and Klavžar and is found useful in determining many degree-based topological indices (listed in Table 1). 
Definition 1. [13] Let $G$ be a graph. Then $M$ - polynomial of $G$ is defined as

$$
M(G ; x, y)=\sum_{i \leq j} m_{i j}(G) x^{i} y^{j}
$$

where $m_{i j}, i, j \geq 1$, is the number of edges $u v$ of $G$ such that $\left\{d_{G}(u), d_{G}(v)\right\}=\{i, j\}$ [14].

Recently, the study of $M$ - polynomial are reported in [15-19].

The Table 1 was given by Deutch and Klavžar to derive degree based topological indices from the $M-$ polynomial.

Table 1. [13] Derivation of some degree-based topological indices from $M$-polynomial.

\begin{tabular}{||cccc||}
\hline Notation & Topological Index & $f(x, y)$ & Derivation from $M(G ; x, y)$ \\
\hline$M_{1}(G)$ & First Zagreb & $x+y$ & $\left.\left(D_{x}+D_{y}\right)(M(G ; x, y))\right|_{x=y=1}$ \\
$M_{2}(G)$ & Second Zagreb & $x y$ & $\left.\left(D_{x} D_{y}\right)(M(G ; x, y))\right|_{x=y=1}$ \\
${ }^{m} M_{2}(G)$ & Second modified Zagreb & $\frac{1}{x y}$ & $\left.\left(S_{x} S_{y}\right)(M(G ; x, y))\right|_{x=y=1}$ \\
$S_{D}(G)$ & Symmetric division index & $\frac{x^{2}+y^{2}}{x y}$ & $\left.\left(D_{x} S_{y}+D_{y} S_{x}\right)(M(G ; x, y))\right|_{x=y=1}$ \\
$H(G)$ & Harmonic & $\frac{2}{x+y}$ & $\left.2 S_{x} J(M(G ; x, y))\right|_{x=1}$ \\
$I_{n}(G)$ & Inverse sum index & $\frac{x y}{x+y}$ & $\left.S_{x} J D_{x} D_{y}(M(G ; x, y))\right|_{x=1}$ \\
$R_{\alpha}(G)$ & General Randić index & $(x y)^{\alpha}$ & $\left.D_{x} D_{y}(M(G ; x, y))\right|_{x=y=1}$ \\
\hline
\end{tabular}

Where $D_{x}=x \frac{\partial f(x, y)}{\partial x}, D_{y}=y \frac{\partial f(x, y)}{\partial y}, S_{x}=\int_{0}^{x} \frac{f(t, y)}{t} d t, S_{y}=\int_{0}^{y} \frac{f(x, t)}{t} d t$ and $J(f(x, y))=f(x, x)$ are the operators.

The Table 2 is given by us in [20] which gives operators to derive general sum connectivity index and the first general Zagreb index from the $M$-polynomial.

Table 2. [20] New operator to derive degree-based topological indices from $M$ - polynomial.

\begin{tabular}{||cccc||}
\hline Notation & Topological Index & $f(x, y)$ & Derivation from $M(G ; x, y)$ \\
$\chi_{\alpha}(G)$ & General sum connectivity [7] & $(x+y)^{\alpha}$ & $\left.D_{x}^{\alpha}(J(M(G ; x, y)))\right|_{x=1}$ \\
$M_{1}^{\alpha}(G)$ & First general Zagreb [21] & $x^{\alpha-1}+y^{\alpha-1}$ & $\left.\left(D_{x}^{\alpha-1}+D_{y}^{\alpha-1}\right)(M(G ; x, y))\right|_{x=y=1}$ \\
\hline
\end{tabular}

Note 1. Hyper Zagreb index is obtained by taking $\alpha=2$ in general sum connectivity index.

Note 2. Taking $\alpha=2,3$ in first general Zagreb index, first Zagreb and forgotten $(F-$ index $)$ topological indices are obtained respectively.

The general Zagreb index or $(a, b)$ - Zagreb index was introduced by Azari et al. [22], which is a generalized version of vertex-degree-based topological index and is defined as

$$
M_{(a, b)}(G)=\sum_{u v \in E(G)}\left(d_{G}(u)^{a} d_{G}(v)^{b}+d_{G}(u)^{b} d_{G}(v)^{a}\right)
$$

The importance of general Zagreb index is that from this index, one can derive seven more topological indices as given in [9]. But in fact, general Zagreb index can also be obtained from the $M$-polynomial with suitable operator, which we present in the Table 3.

Table 3. Operator to derive general Zagreb index from $M-$ polynomial.

\begin{tabular}{||cccc||}
\hline Notation & Topological Index & $f(x, y)$ & Derivation from $M(G ; x, y)$ \\
\hline$M_{(a, b)}(G)$ & General Zagreb index [22] & $x^{a} y^{b}+x^{b} y^{a}$ & $\left.\left(D_{x}^{a} D_{y}^{b}+D_{x}^{b} D_{y}^{a}\right)(M(G ; x, y))\right|_{x=y=1}$ \\
\hline
\end{tabular}

Thus, general Zagreb index can be derived from the $M-$ polynomial. To show this we derive the $M-$ polynomial of cactus graphs in the next section and compare with the results of Nilanjan De obtained in [9]. 


\section{M-Polynomials of cactus chains}

In this section, we obtain $M$ - polynomials of two general cactus chains namely para cacti chain and ortho-cacti chain of cycles. We first compute $M$ - polynomial of para cacti chain of cycles denoted by $C_{m}^{n}$ where $m$ is the length of each cycle and $n$ is the length of the chain. Every block in $C_{m}^{n}$ is a cycle $C_{m}$.

The following theorem gives the $M-$ polynomial of $C_{m}^{n}$.

Theorem 2. Let $C_{m}^{n}$ be para cacti chain of cycles for $m \geq 3, n \geq 2$. Then

$$
M\left(C_{m}^{n} ; x, y\right)=(m n-4 n+4) x^{2} y^{2}+4(n-1) x^{2} y^{4} .
$$

Proof. The para cacti chain of cycles $C_{m}^{n}$ has $m n-n+1$ vertices and $m n$ edges. The edge set of $C_{m}^{n}$ can be partitioned as,

$$
\begin{aligned}
& \left|E_{\{2,2\}}\right|=\mid\left\{u v \in E\left(C_{m}^{n}\right): d_{C_{m}^{n}}(u)=2 \text { and } d_{C_{m}^{n}}(v)=2\right\} \mid=(m n-4 n+4) . \\
& \left|E_{\{2,4\}}\right|=\mid\left\{u v \in E\left(C_{m}^{n}\right): d_{C_{m}^{n}}(u)=2 \text { and } d_{C_{m}^{n}}(v)=4\right\} \mid=4(n-1) .
\end{aligned}
$$

Thus, by using definition of $M-$ polynomial we have,

$$
M\left(C_{m}^{n} ; x, y\right)=(m n-4 n+4) x^{2} y^{2}+4(n-1) x^{2} y^{4} .
$$

Corollary 3. Let $C_{m}^{n}$ be para cacti chain of cycles for $m \geq 3, n \geq 2$. Then

$$
M_{a, b}\left(C_{m}^{n}\right)=2(m n-4 n+4) 2^{a+b}+4(n-1) 2^{a+b}\left(2^{a}+2^{b}\right) .
$$

Proof. To derive general Zagreb index from $M$ - polynomial we use the operator given in Table 3. Now,

$$
\begin{aligned}
M_{a, b}\left(C_{m}^{n}\right)= & \left.\left(D_{x}^{a} D_{y}^{b}+D_{x}^{b} D_{y}^{a}\right)\left(M\left(C_{m}^{n} ; x, y\right)\right)\right|_{x=y=1} \\
= & \left.\left(D_{x}^{a} D_{y}^{b}+D_{x}^{b} D_{y}^{a}\right)\left((m n-4 n+4) x^{2} y^{2}+4(n-1) x^{2} y^{4}\right)\right|_{x=y=1} \\
= & \left(D_{x}^{a} D_{y}^{b}\right)\left((m n-4 n+4) x^{2} y^{2}+4(n-1) x^{2} y^{4}\right) \\
& +\left.\left(D_{x}^{b} D_{y}^{a}\right)\left((m n-4 n+4) x^{2} y^{2}+4(n-1) x^{2} y^{4}\right)\right|_{x=y=1} \\
= & 2(m n-4 n+4) 2^{a+b}+4(n-1) 2^{a+b}\left(2^{a}+2^{b}\right) .
\end{aligned}
$$

The expression obtained above, i.e.,

$$
M_{a, b}\left(C_{m}^{n}\right)=2(m n-4 n+4) 2^{a+b}+4(n-1) 2^{a+b}\left(2^{a}+2^{b}\right)
$$

is same expression obtained in [9], Theorem 1. This shows that the $M$-polynomial has an extra advantage than the general Zagreb index as one can derive about 10 (listed in Tables 1, 2 and 3) degree-based topological indices from the $M$-polynomial including general Zagreb index so far.

The following corollary gives the several topological indices of para cacti chain of cycles derived from $M$-polynomial.

Corollary 4. Let $C_{m}^{n}$ be para cacti chain of cycles for $m \geq 3, n \geq 2$. Then

1. $M_{1}\left(C_{m}^{n}\right)=4 m n+8 n-8$.

2. $M_{2}\left(C_{m}^{n}\right)=4 m n+16 n-16$.

3. ${ }^{m} M_{2}\left(C_{m}^{n}\right)=\frac{m n-2 n+2}{4}$.

4. $S_{D}\left(C_{m}^{n}\right)=2 m n+2 n-2$.

5. $H\left(C_{m}^{n}\right)=\frac{3 m n-8 n-8}{6}$.

6. $I_{n}\left(C_{m}^{n}\right)=\frac{3 m n+4 n-4}{3}$.

7. $\chi_{\alpha}\left(C_{m}^{n}\right)=(m n-4 n+4) 4^{\alpha}+4(n-1) 6^{\alpha}$. 
8. $M_{1}^{\alpha}\left(C_{m}^{n}\right)=(m n-4 n+4) 2^{\alpha}+(n-1) 2^{2 \alpha+1}\left(2^{\alpha-1}+1\right)$.

9. $R_{\alpha}\left(C_{m}^{n}\right)=(m n-4 n+4) 2^{2 \alpha}+(n-1) 2^{3 \alpha+2}$.

Proof. Using the above Theorem 2 and column 4 of Table 1, we get the desired results.

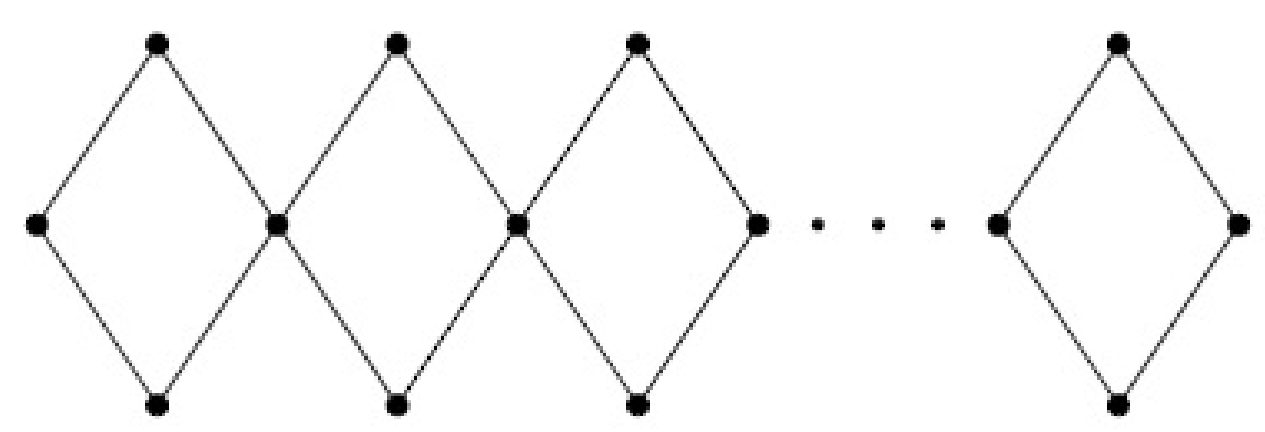

Figure 1. Para-chain square cactus $Q_{n}$

Corollary 5. Let $Q_{n}$ be para-chain square cactus graph for $n \geq 2$. Then

$$
M\left(Q_{n} ; x, y\right)=4 x^{2} y^{2}+4(n-1) x^{2} y^{4}
$$

Proof. Taking $m=4$ in the Theorem 2, we get the desired result.

Corollary 6. Let $Q_{n}$ be para-chain square cactus graph for $n \geq 2$. Then

1. $M_{1}\left(Q_{n}\right)=24 n-8$.

2. $M_{2}\left(Q_{n}\right)=32 n-16$.

3. ${ }^{m} M_{2}\left(Q_{n}\right)=\frac{n+1}{2}$.

4. $S_{D}\left(Q_{n}\right)=10 n-2$.

5. $H\left(Q_{n}\right)=\frac{4 n-8}{6}$.

6. $I_{n}\left(Q_{n}\right)=\frac{16 n-4}{3}$.

7. $\chi_{\alpha}\left(Q_{n}\right)=4^{\alpha+1}+4(n-1) 6^{\alpha}$.

8. $M_{1}^{\alpha}\left(Q_{n}\right)=2^{\alpha+2}+(n-1) 2^{2 \alpha+1}\left(2^{\alpha-1}+1\right)$.

9. $R_{\alpha}\left(Q_{n}\right)=2^{2 \alpha+2}+(n-1) 2^{3 \alpha+2}$.
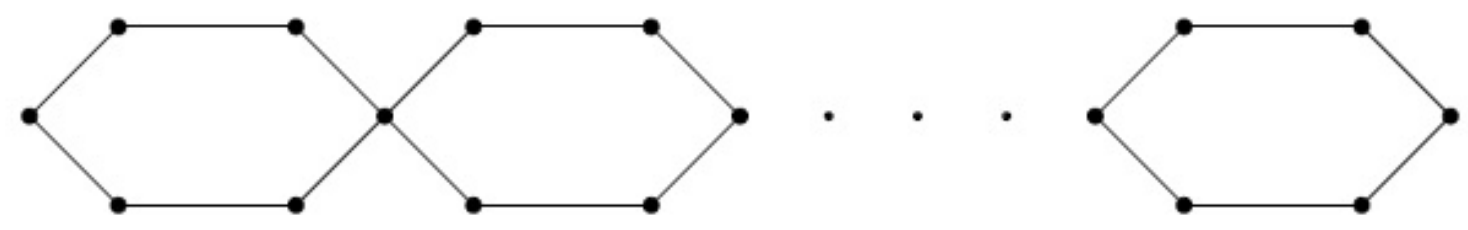

Figure 2. Para-chain hexagonal cactus graph $L_{n}$.

Corollary 7. Let $Q_{n}$ be para-chain square cactus graph for $n \geq 2$. Then

$$
M\left(Q_{n} ; x, y\right)=(2 n+4) x^{2} y^{2}+4(n-1) x^{2} y^{4}
$$

Proof. Taking $m=6$ in the Theorem 2, we get the desired result. 
Corollary 8. Let $L_{n}$ be para-chain hexagonal cactus graph for $n \geq 3$. Then

1. $M_{1}\left(L_{n}\right)=32 n-8$.

2. $M_{2}\left(L_{n}\right)=40 n-16$.

3. ${ }^{m} M_{2}\left(L_{n}\right)=\frac{2 n+1}{2}$.

4. $S_{D}\left(L_{n}\right)=14 n-2$.

5. $H\left(L_{n}\right)=\frac{5 n-4}{3}$.

6. $I_{n}\left(L_{n}\right)=\frac{22 n-4}{3}$.

7. $\chi_{\alpha}\left(L_{n}\right)=(2 n+4) 4^{\alpha}+4(n-1) 6^{\alpha}$.

8. $M_{1}^{\alpha}\left(L_{n}\right)=(2 n+4) 2^{\alpha}+(n-1) 2^{2 \alpha+1}\left(2^{\alpha-1}+1\right)$.

9. $R_{\alpha}\left(L_{n}\right)=(2 n+4) 2^{2 \alpha}+(n-1) 2^{3 \alpha+2}$.

We now consider the ortho-chain cycles with cut-vertices are adjacent. Let $\mathrm{CO}_{m}^{n}$ be ortho-chain cactus graph, where $m$ is the length of each cycle and $n$ is the length of the chain. It is easy to see that $\left|V\left(C O_{m}^{n}\right)\right|=$ $m n-n+1$ and $\left|E\left(C O_{m}^{n}\right)\right|=m n$. In the following theorem we obtain $M$-polynomial of $C O_{m}^{n}$.

Theorem 9. Let $\mathrm{CO}_{m}^{n}$ be ortho cacti chain of cycles for $m \geq 3, n \geq 2$. Then

$$
M\left(C O_{m}^{n} ; x, y\right)=(m n-3 n+2) x^{2} y^{2}+2 n x^{2} y^{4}+(n-1) x^{4} y^{4} .
$$

Proof. The $\mathrm{CO}_{m}^{n}$ be ortho-chain cacti of cycles has $m n-n+1$ vertices and $m n$ edges. The edge partition of $\mathrm{CO}_{m}^{n}$ is given by,

$$
\begin{aligned}
E_{\{2,2\}} & =\left\{u v \in E\left(C O_{m}^{n}\right): d_{C O_{m}^{n}}(u)=2 \text { and } d_{C O_{m}^{n}}(v)=2\right\}, \\
E_{\{2,4\}} & =\left\{u v \in E\left(C O_{m}^{n}\right): d_{C O_{m}^{n}}(u)=2 \text { and } d_{C O_{m}^{n}}(v)=4\right\}, \\
E_{\{4,4\}} & =\left\{u v \in E\left(C O_{m}^{n}\right): d_{C O_{m}^{n}}(u)=4 \text { and } d_{C O_{m}^{n}}(v)=4\right\}, \\
\text { Now, }\left|E_{\{2,2\}}\right| & =m n-3 m+2, \\
\left|E_{\{2,4\}}\right| & =2 n, \\
\left|E_{\{4,4\}}\right| & =n-1 .
\end{aligned}
$$

Thus, the $M-$ polynomial of $C O_{m}^{n}$ is

$$
M\left(C O_{m}^{n} ; x, y\right)=(m n-3 n+2) x^{2} y^{2}+2 n x^{2} y^{4}+(n-1) x^{4} y^{4} .
$$

Corollary 10. Let $C O_{m}^{n}$ be ortho cacti chain of cycles for $m \geq 3, n \geq 2$. Then

1. $M_{1}\left(C O_{m}^{n}\right)=4 m n+8 n-8$.

2. $M_{2}\left(C O_{m}^{n}\right)=4 m n+20 n-24$.

3. ${ }^{m} M_{2}\left(C O_{m}^{n}\right)=\frac{4 m n-7 n+7}{16}$.

4. $S_{D}\left(C O_{m}^{n}\right)=2 m n+n$.

5. $H\left(C O_{m}^{n}\right)=\frac{6 m n-7 n+9}{12}$.

6. $I_{n}\left(C O_{m}^{n}\right)=\frac{3 m n+5 n}{3}$.

7. $\chi_{\alpha}\left(C O_{m}^{n}\right)=(m n-3 n+2) 4^{\alpha}+2 n 6^{\alpha}+(n-1) 8^{\alpha}$.

8. $M_{1}^{\alpha}\left(C O_{m}^{n}\right)=(m n-3 n+2) 2^{\alpha}+(n-1) 2^{2 \alpha-1}+n 2^{\alpha}\left(2^{\alpha-1}+1\right)$.

9. $R_{\alpha}\left(C O_{m}^{n}\right)=(m n-3 n+2) 2^{2 \alpha}+n 2^{3 \alpha+1}+(n-1) 4^{2 \alpha}$.

Proof. Using the Theorem 9 and column 4 of Table 1, we get the desired results.

Now, we consider chain triangular cactus as shown in Figure 3, denoted by $T_{n}$, where $n$ is the length of the $T_{n} . T_{n}$ is special case of $C O_{m}^{n}$ for $m=3$.

Corollary 11. Let $T_{n}$ be the chain triangular cactus for $n \geq 2$. Then

$$
M\left(T_{n} ; x, y\right)=2 x^{2} y^{2}+2 n x^{2} y^{4}+(n-1) x^{4} y^{4} .
$$




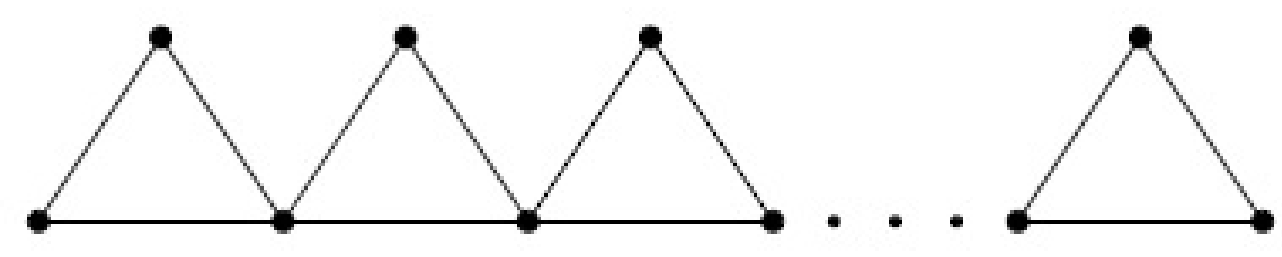

Figure 3. Chain triangular Cactus $T_{n}$.

Proof. The proof follows by substituting $m=3$ in Theorem 9 .

Corollary 12. Let $T_{n}$ be the chain triangular cactus for $n \geq 2$. Then

1. $M_{1}\left(T_{n}\right)=20 n-8$.

2. $M_{2}\left(T_{n}\right)=32 n-24$.

3. ${ }^{m} M_{2}\left(T_{n}\right)=\frac{5 n+7}{16}$.

4. $S_{D}\left(T_{n}\right)=7 n$.

5. $H\left(T_{n}\right)=\frac{11 n+9}{12}$.

6. $I_{n}\left(T_{n}\right)=\frac{14 n}{3}$.

7. $\chi_{\alpha}\left(T_{n}\right)=2^{2 \alpha+1}+2 n 6^{\alpha}+(n-1) 8^{\alpha}$.

8. $M_{1}^{\alpha}\left(T_{n}\right)=2^{\alpha+1}+(n-1) 2^{2 \alpha-1}+n 2^{\alpha}\left(2^{\alpha-1}+1\right)$.

9. $R_{\alpha}\left(T_{n}\right)=2^{2 \alpha+1}+n 2^{3 \alpha+1}+(n-1) 4^{2 \alpha}$.

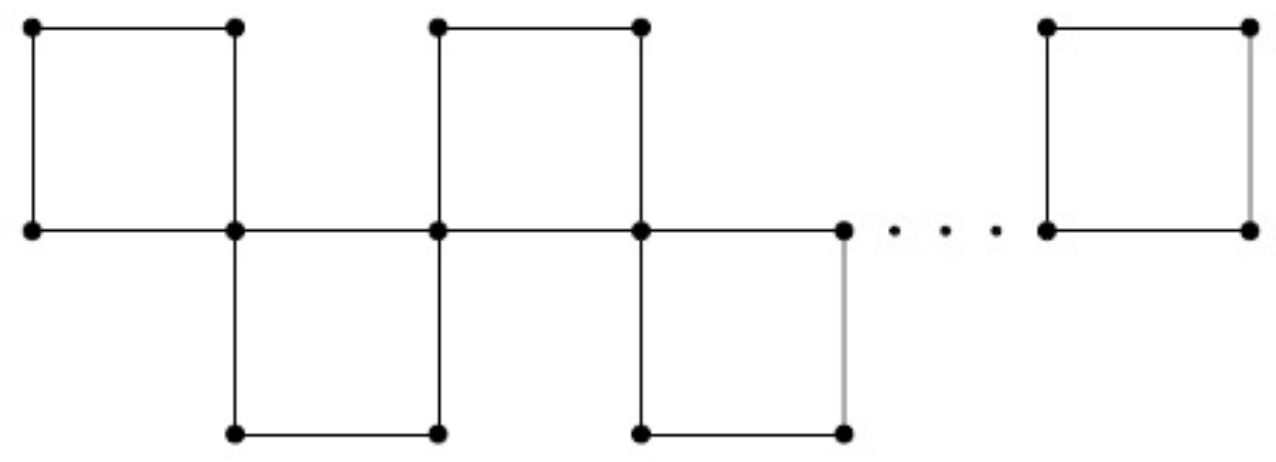

Figure 4. Ortho chain square cactus $O_{n}$.

Corollary 13. Let $O_{n}$ be the ortho-chain square cactus for $n \geq 2$. Then

$$
M\left(O_{n} ; x, y\right)=(n+2) x^{2} y^{2}+2 n x^{2} y^{4}+(n-1) x^{4} y^{4} .
$$

Proof. The proof follows by substituting $m=4$ in Theorem 9 .

Corollary 14. Let $O_{n}$ be the ortho-chain square cactus for $n \geq 2$. Then

1. $M_{1}\left(O_{n}\right)=24 n-8$.

2. $M_{2}\left(O_{n}\right)=36 n-24$. 
3. ${ }^{m} M_{2}\left(O_{n}\right)=\frac{9 n+7}{16}$.

4. $S_{D}\left(O_{n}\right)=9 n$.

5. $H\left(O_{n}\right)=\frac{17 n+9}{12}$.

6. $I_{n}\left(O_{n}\right)=\frac{17 n^{2}}{3}$.

7. $\chi_{\alpha}\left(O_{n}\right)=(n+2) 4^{\alpha}+2 n 6^{\alpha}+(n-1) 8^{\alpha}$.

8. $M_{1}^{\alpha}\left(O_{n}\right)=(n+2) 2^{\alpha}+(n-1) 2^{2 \alpha-1}+n 2^{\alpha}\left(2^{\alpha-1}+1\right)$.

9. $R_{\alpha}\left(O_{n}\right)=(n+2) 2^{2 \alpha}+n 2^{3 \alpha+1}+(n-1) 4^{2 \alpha}$.

The graph $Q(m, n)$ is derived from $K_{m}$ and $m$ copies of $K_{n}$ by identifying every vertex of $K_{m}$ with a vertex of one $K_{n}$ [11]. Here we compute the $M$ - polynomial of the graph $Q(m, n)$ and derive some other topological indices from it. The graph $Q(m, n)$ is depicted in Figure 5.

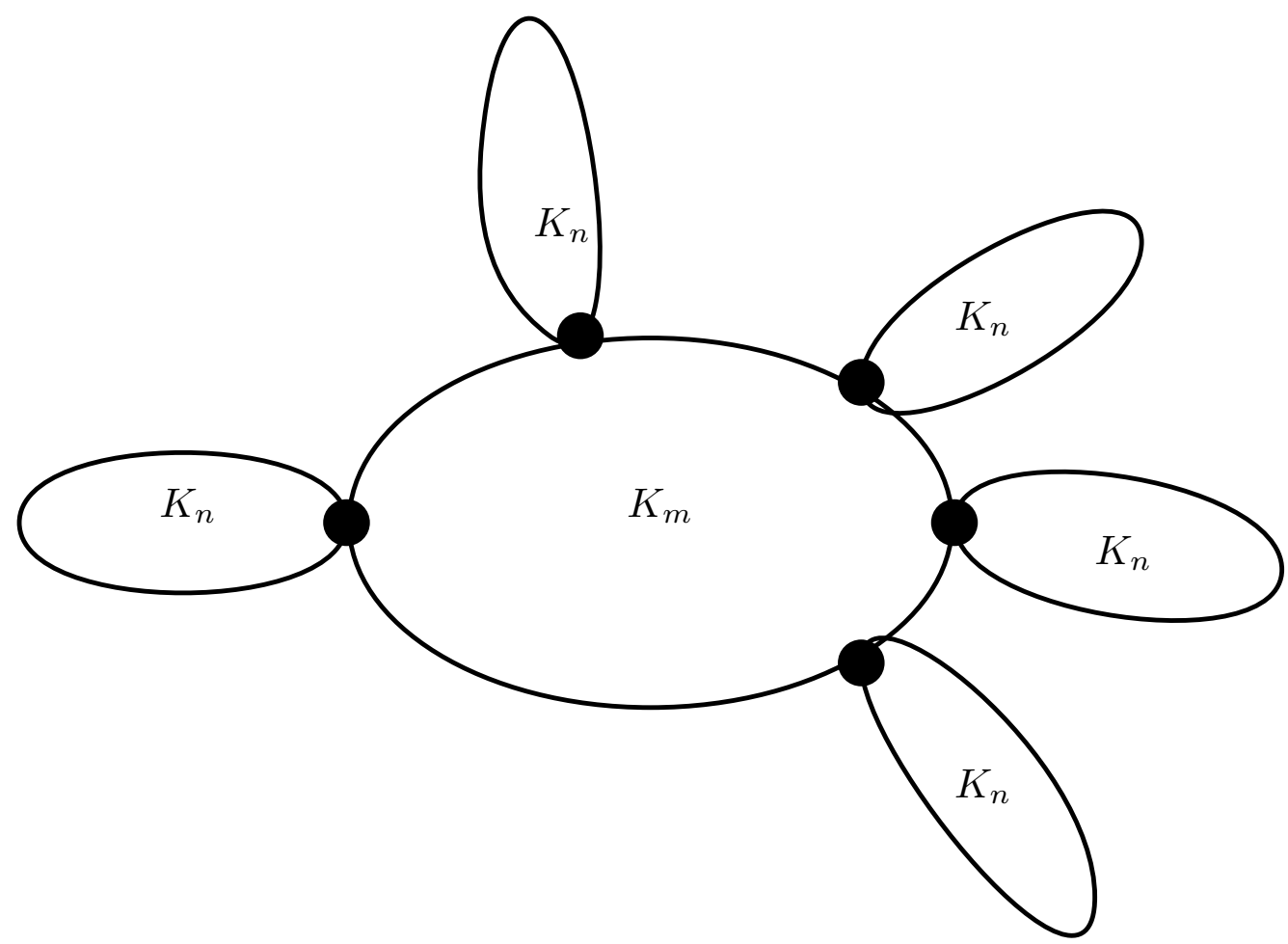

Figure 5. An example of $Q(m, n)$ graph

Theorem 15. Let $Q(m, n)$ be ortho-chain for $m, n \geq 2$. Then

$$
M(Q(m, n) ; x, y)=\frac{m(n-1)(n-2)}{2} x^{n-1} y^{n-1}+m(n-1) x^{n-1} y^{m+n-2}+\frac{m(n-1)}{2} x^{m+n-2} y^{m+n-2} .
$$

Proof. The edge partition of $Q(m, n)$ is partitioned into the following subsets,

$$
\begin{aligned}
E_{1} & =\left\{u v \in E(Q(m, n)): d_{Q(m, n)}(u)=d_{Q(m, n)}(v)=(n-1)\right\}, \\
E_{2} & =\left\{u v \in E(Q(m, n)): d_{Q(m, n)}(u)=(n-1) \text { and } d_{Q(m, n)}(v)=(m+n-2)\right\}, \\
E_{3} & =\left\{u v \in E(Q(m, n)): d_{Q(m, n)}(u)=d_{Q(m, n)}(v)=(m+n-2)\right\}, \\
\text { Now, }\left|E_{1}\right| & =\frac{m(n-1)(n-2)}{2}, \\
\left|E_{2}\right| & =m(n-1), \\
\left|E_{3}\right| & =\frac{m(n-1)}{2} .
\end{aligned}
$$

Thus, the $M$ - polynomial of $Q(m, n)$ is

$$
M(Q(m, n) ; x, y)=\frac{m(n-1)(n-2)}{2} x^{n-1} y^{n-1}+m(n-1) x^{n-1} y^{m+n-2}+\frac{m(n-1)}{2} x^{m+n-2} y^{m+n-2} .
$$


In the para-cacti chain $C_{m}^{n}$, if we join a new vertex and each cycle of length $m \geq 3,\left(C_{m}+K_{1}\right)$ then we call it as wheel chain, denoted by $W_{m}^{n}$. The number of vertices and edges of $W_{m}^{n}$ are $m n+1$ and $2 m n$, respectively. In the following theorem we calculate the $M$-polynomial of $W_{m}^{n}$.

Theorem 16. Let $W_{m}^{n}$ be wheel chain for $m \geq 3, n \geq 2$. Then

$$
M\left(W_{m}^{n} ; x, y\right)=2(m(n-1)-3 n+4) x^{3} y^{3}+4(n-1) x^{3} y^{6}+2(m-1) x^{3} y^{m}+2(n-1) x^{6} y^{m} .
$$

Proof. The edge partition of $W_{m}^{n}$ is partitioned into the following subsets,

$$
\begin{aligned}
E_{1} & =\left\{u v \in E\left(W_{m}^{n}\right): d_{W_{m}^{n}}(u)=d_{W_{m}^{n}}(v)=3\right\}, \\
E_{2} & =\left\{u v \in E\left(W_{m}^{n}\right): d_{W_{m}^{n}}(u)=3 \text { and } d_{W_{m}^{n}}(v)=6\right\}, \\
E_{3} & =\left\{u v \in E\left(W_{m}^{n}\right): d_{W_{m}^{n}}(u)=3 \text { and } d_{W_{m}^{n}}(v)=m\right\}, \\
E_{4} & =\left\{u v \in E\left(W_{m}^{n}\right): d_{W_{m}^{n}}(u)=6 \text { and } d_{W_{m}^{n}}(v)=m\right\}, \\
\text { Now, }\left|E_{1}\right| & =m n-4 n+4, \\
\left|E_{2}\right| & =4(n-1), \\
\left|E_{3}\right| & =m n-2 n+2, \\
\left|E_{4}\right| & =2(n-1) .
\end{aligned}
$$

Thus, the $M$ - polynomial of $W_{m}^{n}$ is

$$
M\left(W_{m}^{n} ; x, y\right)=(m n-4 n+4) x^{3} y^{3}+4(n-1) x^{3} y^{6}+(m n-2 n+2) x^{3} y^{m}+2(n-1) x^{6} y^{m} .
$$

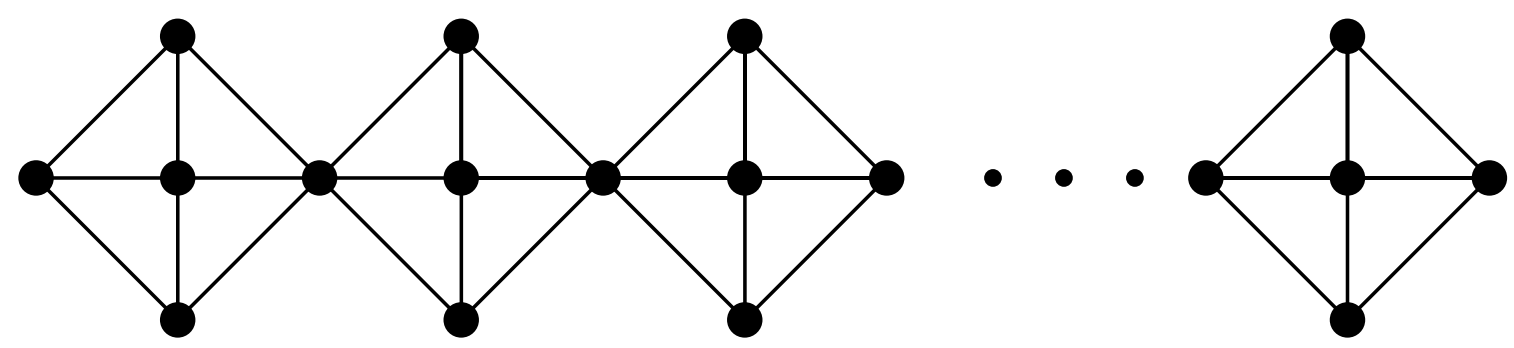

Figure 6. Wheel chain graph $W_{4}^{n}$.

Taking $m=4$ in the Theorem 16, we get the following corollary for $M$ - polynomial of $W_{4}^{n}$. The graph $W_{4}^{n}$ is shown in Figure 6.

Corollary 17. Let $W_{4}^{n}$ be the wheel chain graph for $n \geq 2$. Then

$$
M\left(W_{4}^{n} ; x, y\right)=4 x^{3} y^{3}+4(n-1) x^{3} y^{6}+2(n+1) x^{3} y^{4}+2(n-1) x^{6} y^{4} .
$$

Using Theorem 16 and Tables 1, 2 and 3 one can easily obtain topological indices of wheel chain graph $W_{4}^{n}$. Since it is a routine task, we omit the calculation here.

\section{Conclusion}

In this paper, we have obtained $M$-polynomials of some cactus graphs. We have shown that the general Zagreb index can also be derived from the $M$ - polynomial of a graph. As an example, we have derived general Zagreb index of cactus graphs from the $M$ - polynomial of cactus graphs. The Theorem 1 obtained in [9] is the same as the corollary 3 proved in this paper. Thus generalising the results of general Zagreb index of some cactus graphs. 
Acknowledgments: The authors are thankful to the referee for useful suggestions. The first author is partially supported by the University Grants Commission (UGC), New Delhi, through UGC-SAP-DRS-III for 2016-2021: F.510/3/DRS-III/2016(SAP-I). The second author is supported by University Research Studentship (URS), No.KU/Sch/URS/2017-18/471, dated $3^{\text {rd }}$ July 2018, Karnatak University, Dharwad, Karnataka, India.

Author Contributions: All authors contributed equally to the writing of this paper. All authors read and approved the final manuscript.

Conflicts of Interest: "The authors declare no conflict of interest."

\section{References}

[1] Basavanagoud, B., \& Chitra, E. (2018). On leap Zagreb indices of generalized xyz-point-line transformation graphs $T^{x y z}(G)$ when $z=1$. Mathematical Combinatorics, 2, 44-66.

[2] Basavanagoud, B., \& Chitra, E. (2018). On leap Zagreb indices of some nanostructures. Malaya Journal of Matematik, $6(4), 816-822$.

[3] Basavanagoud, B., \& Chitra, E. (2018). On leap hyper-Zagreb indices of some nanostructures. International Journal of Mathematics Trends and Technology, 64(1), $30-36$.

[4] Basavanagoud, B., \& Jakkannavar, P. (2019). Kulli-Basava Indices of Graphs. International Journal of Applied Engineering Research, 14(1), $325-342$.

[5] Gao, W., Wang, W., Dimitrov, D., \& Wang, Y. (2018). Nano properties analysis via fourth multiplicative ABC indicator calculating. Arabian journal of chemistry, 11(6), 793-801.

[6] Gao, W., Iqbal, Z., Ishaq, M., Aslam, A., \& Sarfraz, R. (2019). Topological aspects of dendrimers via distance based descriptors. IEEE Access, 7, 35619-35630.

[7] Gutman, I. (2013). Degree-based topological indices. Croatica Chemica Acta, 86(4), 351-361.

[8] Li, X., \& Shi, Y. (2008). A survey on the Randić index. MATCH - Communications in Mathematical and in Computer Chemistry, 59(1), 127-156.

[9] De, N. (2019). General Zagreb index of some cactus chains. Open Journal of Discrete Applied Mathematics, $2(1), 24$ - 31.

[10] Sadeghieh, A., Alikhani, S., Ghanbari, N., \& Khalaf, A. J. M. (2017). Hosoya polynomial of some cactus chains. Cogent Mathematics \& Statistics, 4(1), 1305638.

[11] Sadeghieh, A., Ghanbari, N., \& Alikhani, S. (2018). Computation of Gutman index of some cactus chains. Electronic Journal of Graph Theory and Applications, 6(1), 138 - 151.

[12] West, D. B. (1996). An Introduction to Graph Theory. Prentice-Hall: Upper Saddle River, NJ, USA.

[13] Deutsch, E., \& Klavžar, S. (2015). M-Polynomial and degree-based topological indices. Iranian Journal of Mathematical Chemistry, 6(2), $93-102$.

[14] Gutman, I. (2002). Molecular graphs with minimal and maximal Randić indices. Croatica chemica acta, 75(2), $357-369$.

[15] Deutsch, E., \& Klavžar, S. (2019). M-Polynomial Revisited: Bethe Cacti and an Extension of Gutman's Approach. arXiv:1806.08183v2.

[16] Munir, M., Nazeer, W., Rafique, S., \& Kang, S. (2016). M-polynomial and related topological indices of Nanostar dendrimers. Symmetry, 8(9), 97.

[17] Munir, M., Nazeer, W., Nizami, A., Rafique, S., \& Kang, S. (2016). M-polynomials and topological indices of titania nanotubes. Symmetry, 8(11), 117.

[18] Munir, M., Nazeer, W., Rafique, S., \& Kang, S. (2016). M-polynomial and degree-based topological indices of polyhex nanotubes. Symmetry, 8(12), 149.

[19] Basavanagoud, B., \& Jakkannavar, P. M-Polynomial and degree-based topological indices. Electronic Journal of Mathematical analysis and Applications. (Accepted).

[20] Basavanagoud, B., Anand, P. B., \& Praveen, J. M-Polynomial and degree-based topological indices. Submitted for publication.

[21] Li, X., \& Zhao, H. (2004). Trees with the first three smallest and largest generalized topological indices. MATCH Communications in Mathematical and in Computer Chemistry, 50, 57-62.

[22] Azari, M., \& Iranmanesh, A. (2011). Generalized Zagreb index of graphs. Studia Universitatis Babes-Bolyai, Chemia, $56(3), 59-70$.

(C) 2019 by the authors; licensee PSRP, Lahore, Pakistan. This article is an open access article distributed under the terms and conditions of the Creative Commons Attribution (CC-BY) license (http://creativecommons.org/licenses/by/4.0/). 